\title{
Análise exploratória de adoçantes de mesa via espectroscopia no infravermelho (FTIR) e análise por componentes principais (ACP)
}

Exploratory analysis of commercial sweeteners by infrared spectroscopy (FTIR)

and principal component analysis (PCA)

\author{
Adriana TOZETTO ${ }^{1}$, Ivo Mottin DEMIATE ${ }^{1}$, Noemi NAGATA ${ }^{2 *}$
}

\begin{abstract}
Resumo
Nos últimos vinte anos, o consumo de alimentos diet e light tem aumentado sistematicamente, o que tem propiciado o constante desenvolvimento de produtos desse gênero. Grande ênfase tem sido dada àqueles produtos que substituem sacarose por edulcorantes de baixos conteúdos calóricos ou não calóricos. Seguindo esta tendência, adoçantes de mesa têm sido desenvolvidos variando-se amplamente o veículo e o tipo de edulcorante empregado. Neste trabalho, a análise de componentes principais associada à espectroscopia na região do infravermelho médio foi utilizada com sucesso para diferenciar os veículos empregados na produção destes adoçantes, sendo que esta metodologia quimiométrica reduziu o espaço dimensional para dois fatores, explicando cerca de $82 \%$ da variância total dos dados. As variáveis responsáveis por esta discriminação estão localizadas na região da impressão digital do espectro de infravermelho $\left(752,2\right.$ a $\left.1284,5 \mathrm{~cm}^{-1}\right)$. A análise exploratória mostrou-se útil para a visualização destes dados, gerando informações semiquantitativas para os adoçantes constituídos por lactose/aspartame, observações que seriam dificilmente visualizadas sem o recurso quimiométrico aplicado.

Palavras-chave: adoçantes; quimiometria; PCA; FTIR.
\end{abstract}

\begin{abstract}
In the last twenty years, the consumption of diet and light foods has grown steadily, leading to the constant development of such products. Much emphasis has been placed on products that replace sucrose with sweeteners of low or zero calorie content. The development of new commercial sweeteners illustrates this tendency. In this work, principal component analysis and infrared spectroscopy were used to successfully differentiate the vehicles (mediums) employed in the production of sweeteners. This chemometric methodology reduced the dimensional space to two factors, accounting for $82 \%$ of the total variance of the data. The variables responsible for this discrimination were localized in the fingerprint region of the infrared spectrum $\left(752.2\right.$ to $\left.1284.5 \mathrm{~cm}^{-1}\right)$. The exploratory analysis proved effective for visualizing these data, generating semi-quantitative information for the sweeteners composed of lactose/aspartame, which would have been impossible to obtain without the use of the chemometric tool.

Keywords: sweeteners; chemometrics; PCA; FTIR.
\end{abstract}

\section{Introdução}

Nos últimos vinte anos, o consumo de produtos light $\mathrm{e}$ "diet" vem aumentando sistematicamente. Basicamente, estes produtos estão direcionados a pessoas que apresentam algum distúrbio no metabolismo de açúcares (diabéticos) ou, mais recentemente, consumidores que estão em busca de produtos alimentícios de baixo valor calórico ${ }^{16,17}$. A indústria de alimentos geralmente utiliza a mistura de edulcorantes artificiais para propiciar um efeito sinérgico que possibilite empregá-los em menores quantidades, resultando em redução de custo e melhoria de atributos sensoriais ${ }^{5}$. Dentre a ampla variedade de produtos destinados à restrição ou redução de carboidratos existentes para comercialização, destaque deve ser dado aos adoçantes de mesa.

A grande mistura de edulcorantes artificiais e naturais, geralmente empregada neste tipo de amostras, faz com que poucas propostas envolvendo a Espectroscopia no Infravermelho com Transformada de Fourier (FTIR) sejam desenvolvidas ${ }^{2}$. Trata-se de uma técnica espectroscópica de absorção, a qual

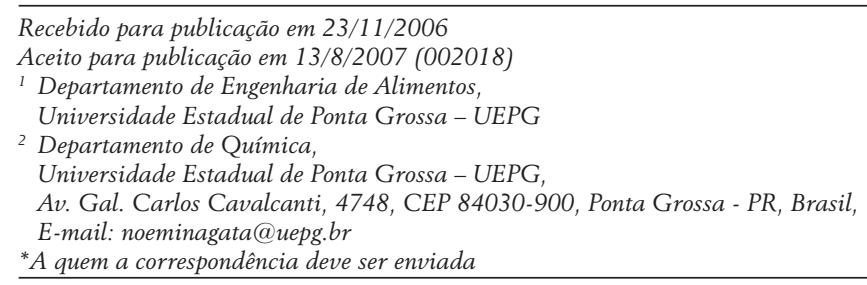

envolve transições moleculares de estados vibracionais ou rotacionais de baixa energia ( 4000 a $400 \mathrm{~cm}^{-1}$ ). Como o número e o tipo de transições que a molécula sofre estão relacionados com o número e o tipo de ligações que ela contém, as informações contidas em espectros deste tipo permitem a identificação de grupos funcionais. Dentro deste contexto, destaque pode ser dado à região da impressão digital (1200 a $700 \mathrm{~cm}^{-1}$ ), que é reconhecida por evidenciar pequenas diferenças na estrutura e constituição da amostra, o que resulta em uma significativa mudança na distribuição dos picos de absorção nesta região do espectro $^{15}$. De qualquer forma, a presença de vários constituintes em uma amostra, aumenta a probabilidade de sobreposição dos sinais de absorção nos espectros de infravermelho.

O avanço computacional e o interfaceamento de instrumentos geram um grande número de variáveis que pode ser medido e analisado para uma única amostra, produzindo uma quantidade enorme de informações. Assim, a avaliação da distribuição de picos em diversas regiões do espectro de infravermelho gera a necessidade de utilização de ferramentas quimiométricas que permitam a compressão de dados sem que haja perda de informação relevante ${ }^{6,11,12}$. A associação entre a espectroscopia no infravermelho e procedimentos quimiométricos, como a Análise por Componentes Principais (ACP), tem recebido muita atenção de pesquisadores de diversas áreas ${ }^{4,7,10,14}$, por se tratar de uma ferramenta analítica rápida que pode detectar diferentes tipos de adulterações em uma única análise. 
Para ilustrar e facilitar a visualização dos principais aspectos envolvidos no ACP, um conjunto constituído por 35 amostras que se distribuem de maneira tridimensional será utilizado (Figura 1). Cada uma destas dimensões pode representar uma variável medida ou, neste caso particular, valores de transmitância registrados em três números de onda. Uma análise mais minuciosa desta ilustração indica que todo o conjunto amostral pode ser delimitado por um tetraedro regular, cuja maior particularidade está representada por uma pequena altura.

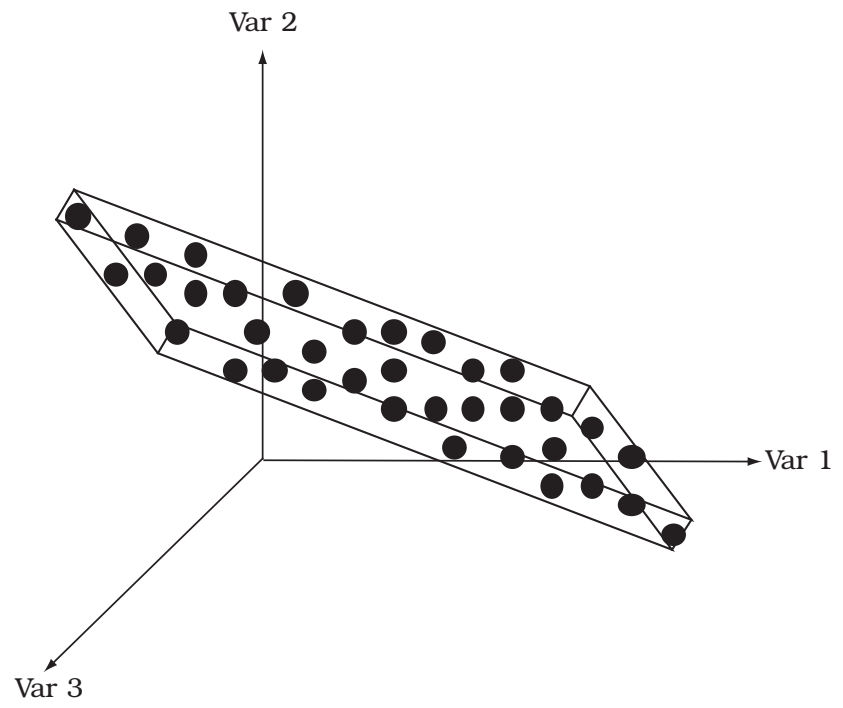

Figura 1. Gráfico tridimensional do conjunto de dados composto por 35 amostras.

Em função deste aspecto, e principalmente da característica mais fundamental do ACP (redução do espaço dimensional), uma rotação e/ou transformação dos eixos originais é realizada. Este novo sistema de eixos (mais comumente denominados como fatores, componentes principais, ou ainda variáveis latentes) apresenta a direção da máxima variância dos dados, conforme pode ser observado na Figura $2 \mathrm{a}^{9}$.

A primeira componente principal (CP1) tem a direção que descreve o máximo espalhamento das amostras, sendo responsável pela explicação de grande parte da variância apresentada pelo conjunto. No entanto, apenas esta componente principal não é suficiente para explicar o comportamento global deste conjunto de dados, sendo necessária uma segunda componente (CP2), a qual deve ser ortogonal à primeira e responsável pela explicação de uma parcela importante da variância observada. Em princípio, é possível extrair tantas componentes principais quanto o número de variáveis. No entanto, devido à baixa variabilidade dos dados em torno da terceira componente principal (CP3), é possível que esta seja descartada. Este procedimento atende às duas grandes premissas do ACP, ou seja, a redução de variáveis, sem perda de informação relevante ${ }^{9,11,12}$.

Em termos analíticos instrumentais, esta redução do espaço dimensional, conseguido através da seleção de poucas componentes principais, possibilita remover o ruído instrumental (ou variações aleatórias), bem como as informações redundantes fornecidas por variáveis altamente correlacionadas (colinearidade).

Um grande número de trabalhos tem reportado a utilização de ACP associado à espectroscopia no infravermelho médio no estudo de matrizes de interesse alimentício. LAI, KEMSLEY e WILSON $^{13}$ investigaram o potencial desta técnica associada à análise discriminante para verificar a autenticidade de óleos vegetais, tais como, azeite de oliva refinado e azeite de oliva extravirgem. Embora estas duas amostras de interesse sejam quimicamente e espectroscopicamente muito semelhantes,
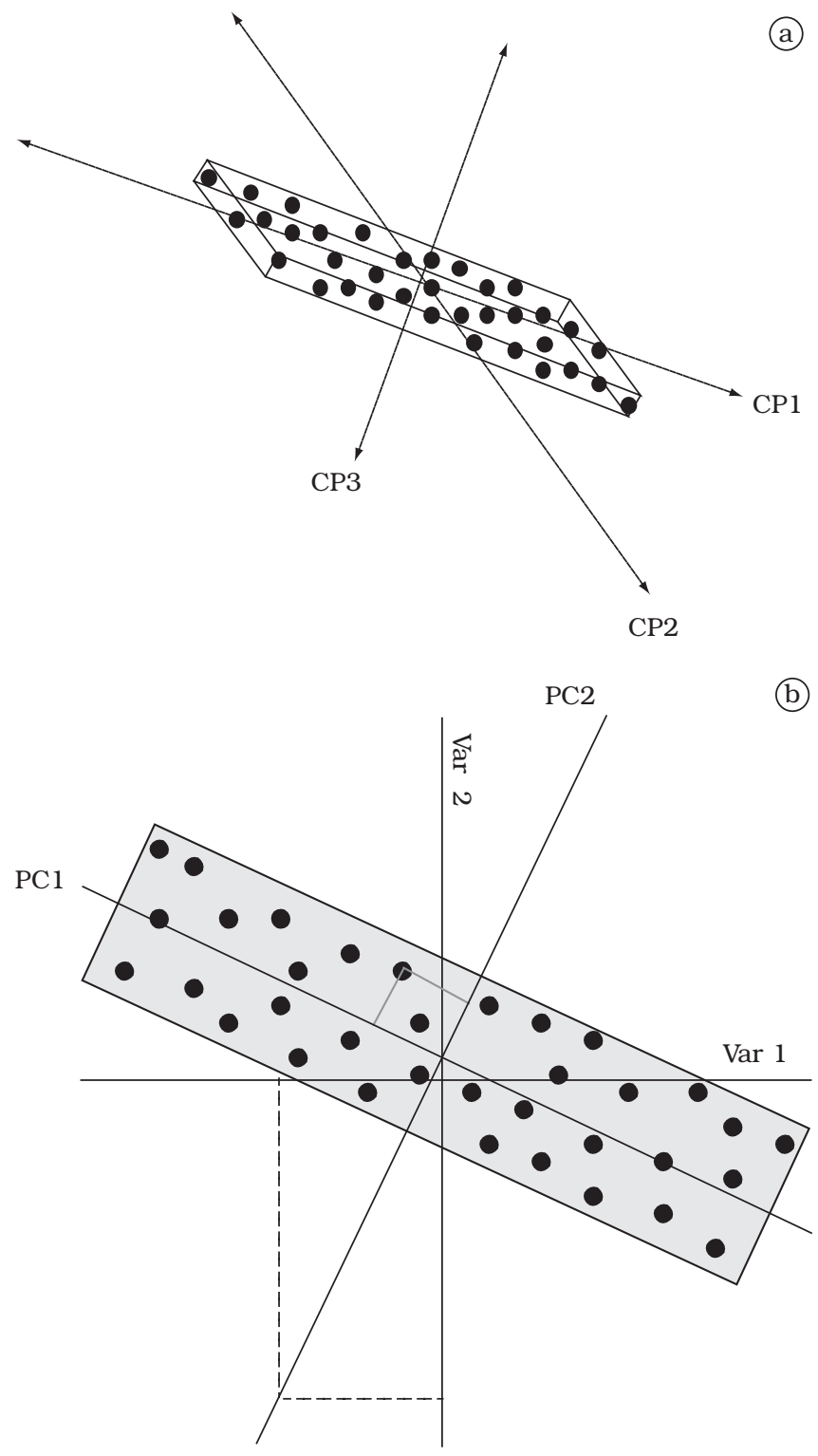

Figura 2. a) Gráfico tridimensional ilustrando os eixos das componentes principais; e b) Gráfico bidimensional da CP1 versus. CP2: escores representados por ( $\longrightarrow$ ) e pesos por (-----).

a análise multivariada gerou agrupamentos diferenciados, possibilitou a discriminação correta destes dois tipos de óleos e demonstrou seu potencial para a rápida identificação de adulterantes nestes produtos. ZAGONEL, PERALTA-ZAMORA e RAMOS $^{18}$, desenvolveram um procedimento para monitorar a 
etanólise em óleo de soja degomado. Os reagentes (triglicerídeos) e os produtos (etil ésteres) envolvidos na etanólise possuem um espectro médio no infravermelho muito semelhante. Porém, quando os espectros derivados foram tratados pelo ACP, na região entre $1700-1800 \mathrm{~cm}^{-1}$, a segunda componente principal propiciou uma indicação do andamento da reação estudada. AL-JOWDER, KEMSLEY, WILSON ${ }^{1}$ discriminaram carne de boi contendo $20 \%$ de adulterantes (coração, tripa, rim, e fígado) via FTIR-ATR. Foram obtidos bons resultados, sendo que o regime de cozimento mostrou dificultar esta identificação. DUPUY et al. ${ }^{8}$ utilizaram FTIR-ATR, na faixa espectral entre 1500 a $700 \mathrm{~cm}^{-1}$, para desenvolver um método de discriminação de amido de milho modificado. Apesar do grande potencial da análise por ACP, a semelhança entre os dados espectroscópicos propiciou apenas uma discriminação parcial dos amidos em subgrupos.

Em função dos prospectos positivos, o presente trabalho tem o objetivo de realizar um estudo preliminar das potencialidades da ACP nos dados espectroscópicos de adoçantes de mesa comerciais. Esta análise exploratória permitirá avaliar a potencialidade da técnica espectroscópica na obtenção de uma metodologia rápida que evidencie a presença dos edulcorantes, mesmo que estes estejam presentes em baixas concentrações nas amostras de interesse.

\section{Material e métodos}

\subsection{Material}

Os adoçantes de mesa foram obtidos nos supermercados locais, sendo priorizadas as amostras comercializadas na forma sólida (pó fino). A Tabela 1 apresenta a composição informada pelo fabricante. Para a análise destas amostras via FTIR, foram preparadas pastilhas contendo cerca de $1 \mathrm{mg}$ do adoçante e 100 mg de $\mathrm{KBr}$ (grau espectroscópico IR Merck). Para tal, processos de homogeneização e trituração da amostra e $\mathrm{KBr}$ foram realizados com o auxílio de um almofariz e pistilo de ágata. Posteriormente, toda a massa foi transferida cuidadosamente para o pastilhador onde uma pressão de 60 toneladas. $\mathrm{cm}^{-2}$ foi aplicada para a confecção da pastilha. As pastilhas foram secas a $60{ }^{\circ} \mathrm{C}$ por 6 horas, garantindo que os edulcorantes com instabilidade a altas temperaturas não fossem degradados.

\subsection{Métodos}

A análise de todas as amostras foi realizada em um espectrofotômetro de infravermelho Shimadzu, modelo FTIR 8.400, na região espectral entre 4000 a $400 \mathrm{~cm}^{-1}$, com resolução de $1,93 \mathrm{~cm}^{-1}$. Antes da coleta dos espectros das amostras, um espectro da pastilha do branco (contendo apenas $100 \mathrm{mg}$ de $\mathrm{KBr}$ ) foi realizado para correção de ruídos instrumentais.

\subsection{Análise multivariada}

O espectro FTIR (contendo um total de 1866 pontos) das 21 amostras de adoçantes de mesa comerciais (Tabela 1), contendo diferentes veículos (sacarose, lactose, maltodextrina e dextrose) e edulcorantes (aspartame, sacarina, ciclamato, esteviosídeo, sorbitol, sucralose, acesulfame-K) foram processados via análise de componentes principais (ACP). Os dados espectrais foram transportados em formato ASCII pelo programa Microcal Origin $^{\mathrm{TM}}$ (Versão 5.0) e a análise multivariada dos dados foi realizada empregando-se o programa Pirouette for Windows 3.11 .

Tabela 1. Composição declarada pelos fabricantes das amostras comerciais de adoçantes.

\begin{tabular}{cll}
\hline Amostra & \multicolumn{1}{c}{ Veículo } & \multicolumn{1}{c}{ Edulcorante artificial } \\
\hline 1 & Lactose $(93,75 \%)$ & Aspartame \\
2 & Lactose $(93,75 \%)$ & Aspartame \\
3 & Lactose $(95,7 \%)$ & Aspartame \\
4 & Lactose $(95,4 \%)$ & Aspartame \\
5 & Lactose $(95,0 \%)$ & Aspartame \\
6 & Lactose & Ciclamato, Sacarina e \\
& & Esteviosídeo \\
7 & Lactose & Ciclamato, Sacarina e \\
& & Esteviosídeo \\
8 & Lactose & Esteviosídeo \\
9 & Lactose & Ciclamato, Sacarina, \\
& & Aspartame e Acesulfame-K \\
10 & Lactose e Maltodextrina & Aspartame e Acesulfame-K \\
11 & Lactose e Maltodextrina & Ciclamato, Sacarina e \\
& & Esteviosídeo \\
12 & Sacarose & Sulcralose \\
13 & Sacarose & Sulcralose \\
14 & Sacarose & Aspartame \\
15 & Sacarose & Ciclamato e Sacarina \\
16 & Sacarose & Ciclamato, Sacarina e \\
& & Aspartame \\
17 & Maltodextrina & Esteviosídeo \\
18 & Maltodextrina & Ciclamato e Sacarina \\
19 & Maltodextrina & Ciclamato e Sacarina \\
20 & Maltodextrina & Sorbitol, Ciclamato e Sacarina \\
21 & Dextrose e Maltodextrina & Aspartame \\
\hline & &
\end{tabular}

\section{Resultados e discussão}

As amostras deste estudo possuem estruturas semelhantes e muitas vezes grupos funcionais iguais. Esta semelhança estrutural e a mistura resultante encontrada nos produtos comerciais fazem com que o espectro FTIR dos adoçantes comerciais seja muito semelhante. Estas características impossibilitam a diferenciação dos adoçantes apenas pela análise visual dos espectros. A Figura 3 mostra a grande quantidade de informação similar (mesmo para amostras com diferentes veículos) que os espectros FTIR de adoçantes comerciais possuem o que evidencia a necessidade de uma ferramenta que possibilite analisar qualitativamente este tipo de amostras.

A Análise por Componentes Principais (ACP) foi a ferramenta analítica empregada com o intuito de diferenciar os adoçantes de mesa de acordo com a sua composição, ou seja, certificar e caracterizar a presença dos edulcorantes naturais e artificiais. Esta análise foi realizada inicialmente pelo processamento dos dados da transmitância de toda faixa espectral do infravermelho (4000 a $400 \mathrm{~cm}^{-1}$, conforme mostra a Figura 3) utilizando o programa Pirouette for Windows ${ }^{\mathrm{TM}} 3.11$. Foram testados os dados originais e alguns tipos de transformações de 


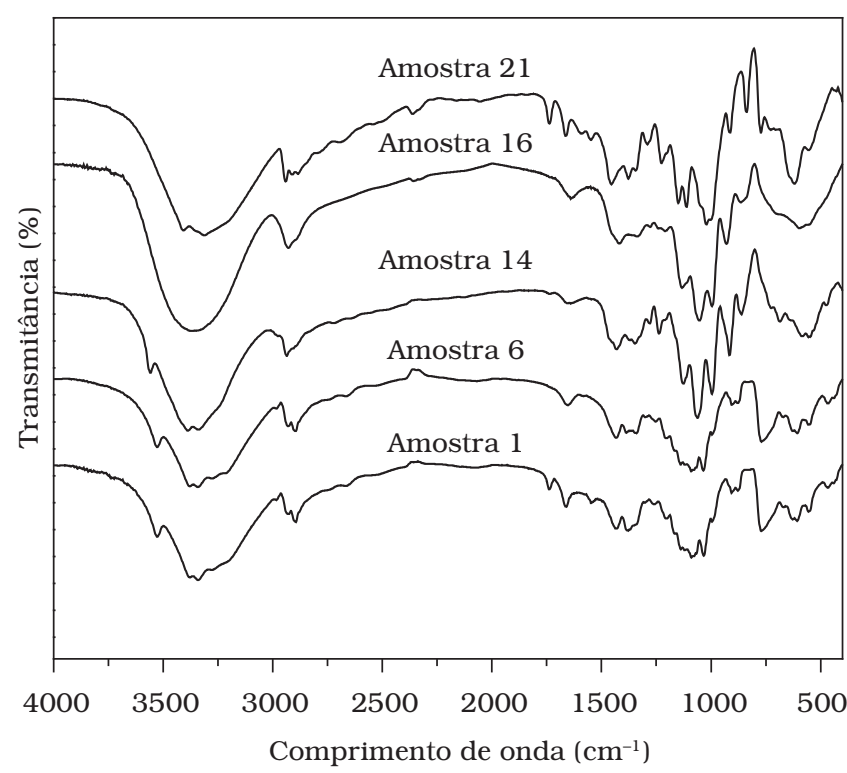

Figura 3. Espectro de FTIR ( 400 a $4000 \mathrm{~cm}^{-1}$ ) de algumas amostras de adoçantes comerciais.

dados ( $1^{\text {a }}$ e $2^{\text {a }}$ derivada) concomitantemente ao procedimento de pré-processamento com dados centrados na média.

A melhor discriminação para os adoçantes de mesa foi obtida pela aplicação da $1^{\text {a }}$ derivada aos dados espectrais. Geralmente, este procedimento realiza uma espécie de deconvolução parcial de sinais não totalmente sobrepostos, o que possibilita que pequenas diferenças sejam melhor evidenciadas por este tipo de transformação. A ACP mostrou que duas componentes principais são responsáveis pela distinção entre os veículos. A Primeira Componente Principal (CP1) descreve 62,53\% da variância dos dados, enquanto a Segunda Componente Principal (CP2) 19,67\%, totalizando 82,21\%. A análise de escores da CP1 versus CP2 (Figura 4) apresenta uma clara separação das amostras pelos veículos que as compõem.

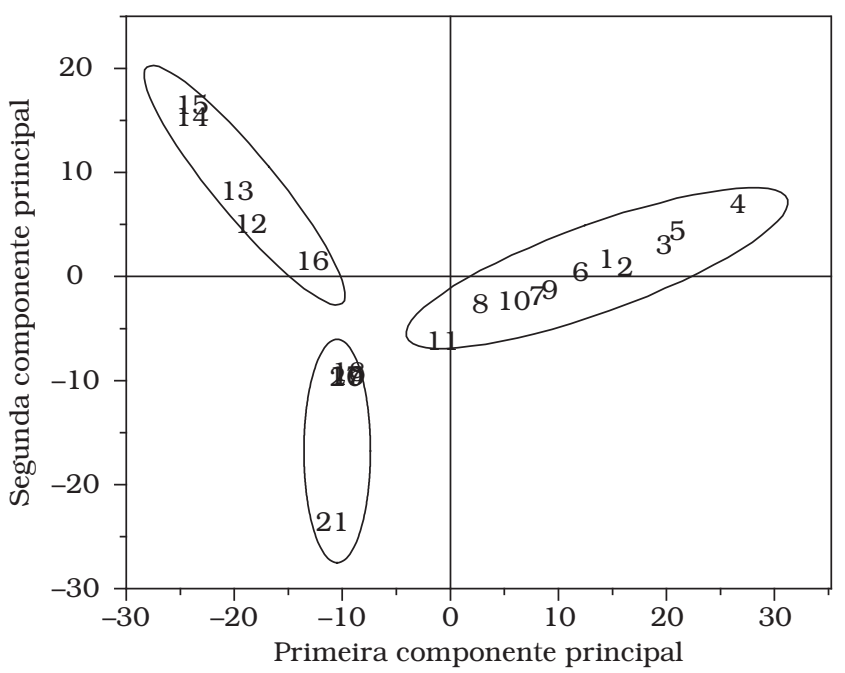

Figura 4. Gráfico de escores do ACP na análise de adoçantes de mesa via FTIR.
Pela análise de escores é possível observar que para altos valores de CP1 estão as amostras de adoçantes de mesa que possuem o veículo lactose $(1,2,3,4,5,6,7,8$ e 9) e para baixos valores de CP1 apresentam-se as amostras com veículo sacarose $(12,13,14,15$ e 16) e maltodextrina $(17,18,19$, 20 e 21).

Enquanto isso, a CP2 propicia duas outras separações, a dos outros veículos (sacarose e maltodextrina) e também uma que envolve a participação de edulcorantes utilizados para a produção dos adoçantes de mesa. Na primeira, as amostras com veículo sacarose agrupam-se na região positiva da CP2, enquanto inversamente as amostras com veículo maltodextrina na região negativa da CP2. A segunda separação, propiciada pela CP2, é menos evidente e retrata diferenciações apenas para as amostras que contêm o veículo lactose (amostras 1 a 11 , localizadas na parte positiva da CP1). Para altos valores de CP2 encontram-se as amostras que contêm aspartame como único edulcorante artificial na composição (amostras 1 a 5). Por outro lado, para baixos valores de CP2 encontram-se os adoçantes de mesa constituídos por vários edulcorantes artificiais (amostras 6 a 12).

As amostras 10 e 11 não foram citadas anteriormente, pois possuem uma particularidade: ambas contêm na sua composição dois diferentes veículos, lactose e maltodextrina. A localização destas amostras em termos de escores mostra que elas não apresentam nem valores muito positivos (das amostras contendo como veículo apenas lactose) e nem negativos (das amostras contendo como veículo apenas maltodextrina) na CP1, indicando que o veículo deve ter realmente uma composição mista.

Uma tendência similar ocorre com a amostra 21, que tem como veículo uma mistura de maltodextrina e dextrose. Em função das diferenças de composição, enquanto as amostras que possuem exclusivamente maltodextrina aparecem agrupadas em valores negativos de CP1 e CP2, a amostra 21, particularmente, aparece deslocada no sentido de valores mais negativos de CP2 que as demais amostras.

A discriminação obtida via ACP está relacionada principalmente aos diferentes tipos de veículos presentes nos adoçantes de mesa comerciais e não aos edulcorantes utilizados na sua fabricação. Isto ocorre porque o espectro FTIR dos adoçantes corresponde ao espectro de uma mistura, veículo + edulcorantes. Como o veículo é o componente em maior quantidade (corresponde a mais de $90 \%$ do conteúdo total em todas as amostras) o espectro é muito semelhante ao espectro do veículo puro. Este resultado não indica a falta de representatividade dos edulcorantes, mas sim das suas baixas concentrações e da alta sobreposição espectral devido à combinação de vários edulcorantes.

Uma vez que os escores correspondem às coordenadas das amostras no espaço definido pelas novas componentes principais, enquanto que os pesos representam a importância de cada variável original no processo de combinação linear que permite o surgimento destas componentes, a análise conjunta de ambos os argumentos permite evidenciar as regiões espectrais que viabilizam a discriminação observada (Figura 4). Vários 
comprimentos de onda apresentam valores de peso próximos a zero, sendo que a região espectral de maior importância para a discriminação das amostras (valores altamente positivos e negativos de pesos) se localiza entre 752,19 e 1284,5 cm-1 (Figura 5), que corresponde à região da impressão digital.
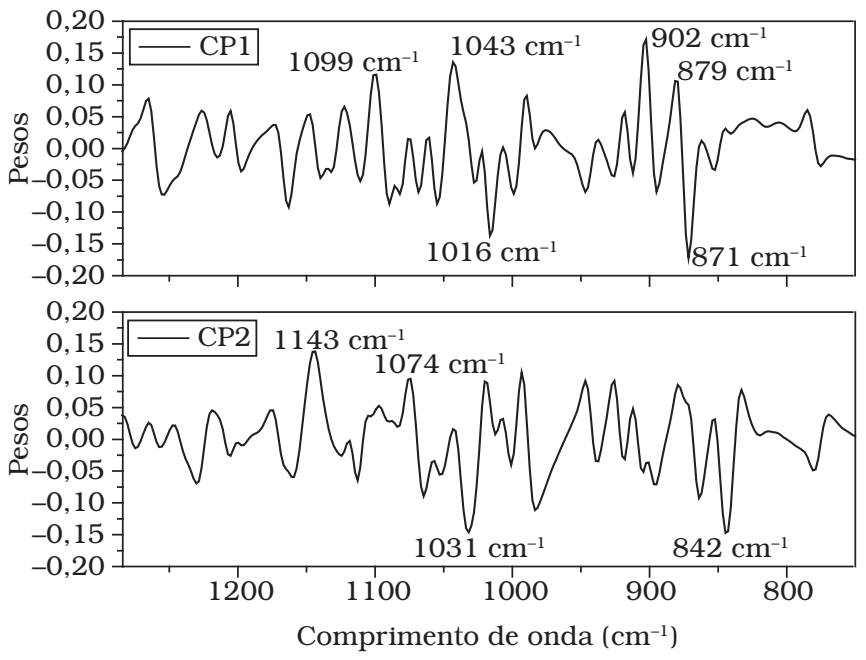

Figura 5. Gráfico de pesos para as duas primeiras componentes principais.

Analisando o gráfico de escores, Figura 4, observa-se claramente que todas as amostras que possuem o veículo lactose em sua composição encontram-se em altos valores positivos de CP1. Através da análise dos pesos (Figura 5), verifica-se que os valores mais positivos de peso para a CP1 ocorrem em sinais localizados nos comprimentos de onda $902,62 \mathrm{~cm}^{-1}$ (alto valor positivo de peso $=0,2016), 1043,42 \mathrm{~cm}^{-1}$ (peso $\left.=0,1583\right)$, $1099,35 \mathrm{~cm}^{-1}$ (peso $\left.=0,1305\right)$ e $879,48 \mathrm{~cm}^{-1}$ (peso $=0,1108$ ). Estes sinais estão presentes no espectro derivado da lactose pura que representam os pontos de inflexão significativos do seu espectro original (Figura 6).

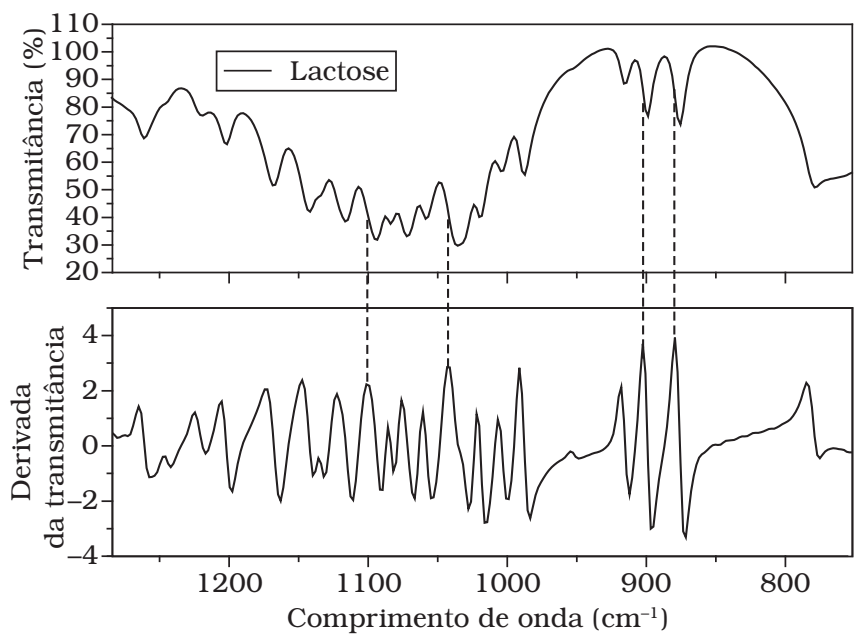

Figura 6. Espectro FTIR original e derivado da lactose pura.

Por outro lado, as amostras que apresentam em sua composição o veículo sacarose ou maltodextrina encontram-se em valores negativos de CP1. Observando-se o gráfico de pesos, verifi- ca-se que os valores mais negativos de peso para a CP1 ocorrem em um sinal localizado no comprimento de onda $871,76 \mathrm{~cm}^{-1}$ (alto valor negativo de peso $=-0,2020$ ), seguido pelo sinal em $1016,41 \mathrm{~cm}^{-1}$ (valor negativo de peso $=-0,1612$ ). Isso indica que a ausência de sinais característicos da lactose e a presença de um ponto de inflexão na região de 871,76 e $1016,41 \mathrm{~cm}^{-1}$ separam os veículos que compóem os adoçantes de mesa.

Em relação a CP2, é possível observar um comportamento diferenciado da amostra 21, que se localiza nas regiões mais negativas desta CP. Este aspecto é provavelmente decorrente da presença de dextrose com pontos de inflexão característicos nos comprimentos de onda $842,83 \mathrm{~cm}^{-1}$ e $1031,85 \mathrm{~cm}^{-1}$, sinais estes que apresentam valor de peso negativo na $\mathrm{CP} 2$, sendo $-0,1816$ e $-0,1649$, respectivamente.

A segunda distinção possível de ser realizada analisando-se a CP2 está relacionada ao agrupamento deslocado na região positiva de CP1. Uma pequena diferença pode ser observada entre as amostras compostas basicamente por lactose/aspartame (se localizam na região positiva de $\mathrm{CP} 2$ ) daquelas compostas por lactose/mistura de edulcorantes (tendendo à região negativa de CP2). Este deslocamento para regióes positivas de CP2 das amostras contendo apenas lactose/aspartame pode ser associado aos pesos (Figura 5) que indicam que os valores mais positivos de peso para a CP2 ocorrem em sinais localizados nos comprimentos de ondas $1143,42 \mathrm{~cm}^{-1}$ (alto valor positivo de peso $=0,1530$ ) e $1074,28 \mathrm{~cm}^{-1}$ (peso $\left.=0,1078\right)$. Estes pontos de inflexão estão presentes no espectro do aspartame puro, conforme mostra a Figura 7.

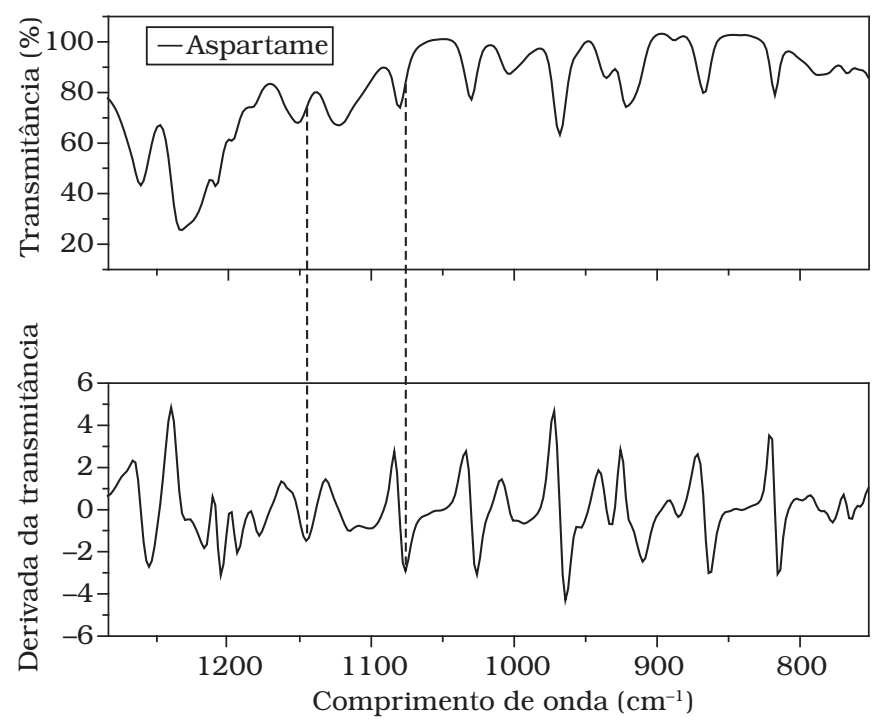

Figura 7. Espectro FTIR original e derivado do aspartame puro.

Associando-se as informações cedidas por alguns fabricantes de adoçantes de mesa e a análise deste modelo multivariado é possível inferir dados para uma análise semiquantitativa da concentração do veículo lactose presente em algumas destas amostras. O conjunto das amostras com composição lactose/aspartame pode ser subdivido em dois subgrupos 
(Figura 4; subgrupo 1: amostras 3, 4 e 5; subgrupo 2: amostras 1 e 2). Uma análise mais detalhada indica que as amostras com maior concentração de lactose (subgrupo 1, com cerca de 95\% de lactose) estão deslocadas para regiões mais positivas de CP1 e CP2, quando comparadas ao subgrupo 2 que contém $93,75 \%$ de lactose.

Em função dos resultados apresentados é possível observar que, quando associada a processos de calibração multivariada, a técnica espectroscópica (FTIR) apresenta grande potencial para discriminação de amostras deste tipo, mesmo em situações de estreita semelhança composicional. Adicionalmente, os resultados sugerem a possibilidade de realização de uma análise quantitativa para os componentes majoritários das amostras estudadas, observação esta que é corroborada em trabalhos publicados na literatura recente envolvendo matrizes similares $^{2,3}$.

\section{Conclusão}

O trabalho ressalta a importância na utilização de metodologia quimiométrica ( $\mathrm{ACP}$ ) no tratamento de dados obtidos via espectroscopia no infravermelho para a análise exploratória de adoçantes de mesa. A utilização de ACP permitiu uma ótima discriminação para os veículos que compõem os adoçantes de mesa, além de proporcionar indícios de uma promissora análise semiquantitativa do veículo lactose.

Uma vez que os veículos apresentam altas concentrações nas amostras de interesse, uma análise pormenorizada dos edulcorantes artificiais ficou prejudicada.

\section{Agradecimentos}

Os autores agradecem à Fundação Araucária - PR/Apoio à Instalação de Recém-Doutores (Chamada 04/01 - Protocolo 1017) pelo apoio financeiro.

\section{Referências bibliográficas}

1. AL-JOWDER, O.; KEMSLEY, E. K.; WILSON, R. H. Detection of adulteration in cooked meat products by mid-infrared spectroscopy. Journal of Agricultural and Food Chemistry, v. 50, n. 6, p. 1325-1329, 2002.

2. ARMENTA, S.; GARRIGUES, S.; DE LA GUARDIA, M. FTIR Determination of Aspartame and Acesulfame-K in Tabletop Sweeteners. Journal of Agricultural and Food Chemistry, v. 52, n. 26, p. 7798-7803, 2004.

3. ARMENTA, S.; GARRIGUES, S.; DE LA GUARDIA, M. Sweeteners Determination in Table Top Formulations Using FT-Raman Spectrometry and Chemometric Analysis. Analytica Chimica Acta, v. 521, n. 2, p. 149-155, 2004.
4. BORIN, A.; POPPI, R. J. Multivariate quality control of lubricating oils using Fourier transform infrared Spectroscopy. Journal Brazilian Chemical Society, v. 15, n. 4, p. 570-576, 2004.

5. CAPITAN-VALLVEY, L. F. et al. Resolution of an intense sweetener mixture by use of a flow injection sensor with on-line solid-phase extraction. Analytical and Bioanalytical Chemistry, v. 385, n. 2, p. 385-391, 2006.

6. COCCHI, M. et al. Use of multivariate analysis of MIR spectra to study bread staling. Annali di Chimica, v. 95, n. 9-10, p. 657-666, 2005.

7. COTRIM, A. R. et al. Identifying the origin of lignins and monitoring their structural changes by means of FTIR-PCA and-SIMCA. Bioresource Technology, v. 68, n. 1, p. 29-34, 1999.

8. DUPUY, N. et al. Mid-infrared spectroscopy and chemometrics in corn starch classification. Journal of Molecular Structure, v. 410-411, p. 551-554, 1997.

9. FERREIRA, M. M. C. et al. Quimiometria I: calibração multivariada, um tutorial. Química Nova, v. 22, n. 5, p. 724-731, 1999.

10. FOOT, M.; MULHOLLAND, M. Classification of chondroitin sulfate A, chondroitin sulfate $\mathrm{C}$, glucosarnine hydrochloride and glucosamine 6 sulfate using chernometric techniques. Journal of Pharmaceutical and Biomedical Analysis, v. 38, n. 3, p. 397-407, 2005.

11. HOPKE, P. K. The evolution of chemometrics. Analytica Chimica Acta, v. 500, n. 1-2, p. 365-377, 2003.

12. KATEMAN, G.; BUYDENS, L. Quality Control in Analytical Chemistry - A Series of Monographs on Analytical Chemistry and Its Applications, $2^{\text {a }}$ edição. New York: Wiley, 1993.

13. LAI, Y. W.; KEMSLEY, K. E.; WILSON, R. H. Potential of Fourier transform-infrared spectroscopy for the authentication of vegetable-oils. Journal of Agricultural and Food Chemistry, v. 42, n. 5, p. 1154-1159, 1994.

14. MARIEY, L. et al. Discrimination, classification, identification of microorganisms using FTIR spectroscopy and chemometrics. Vibrational Spectroscopy, v. 26, n. 2, p. 151-159, 2001.

15. MIRANDA, T. M. R.; Gonçalves, A. R.; Pessoa Amorim, M. T. Ultraviolet-induced crosslinking of poly (vinyl alcohol) evaluated by principal component analysis of FTIR spectra. Polymer International, v. 50, n. 10, p. 1068-1072, 2001.

16. NELSON, L. A.; Sweetners: Alternative, USA: Eagan Press, 1999.

17. PINHEIRO, M. V. S. et al. The effect of different sweeteners in low-calorie yogurts - a review. International Journal of Dairy Technology, v. 58, n. 4, p. 193-199, 2005.

18. ZAGONEL, G. F.; PERALTA-ZAMORA, P.; RAMOS, L. P. Multivariate monitoring of soybean oil ethanolysis by FTIR. Talanta, v. 63 , n. 4 , p. 1021-1025, 2004. 